\title{
Probability Model of Power Equipment Maintenance Based on Full State Process
}

\author{
Yantao Wang ${ }^{1,2, *}$ and Xueshan Han $^{1}$ \\ ${ }^{I}$ Key Laboratory of Power System Intelligent Dispatch and Control of Ministry of Education, Shandong University, Ji- \\ nan 250061, China \\ ${ }^{2}$ School of Economics and Management, Northeast Dianli University, Jilin 132012, China
}

\begin{abstract}
Equipment maintenance is one of the core tasks in power system operations. The premise and foundation for maintenance is to establish a power equipment maintenance model, which should reflect all equipment states accurately including operation, inspection and maintenance. On the basis of the analysis of the traditional state diagram probability model, an improvement is put forward to revise the model for keeping in line with the actual operation and maintenance situation of power equipment. The effectiveness and practicability of the improved model are validated perfectly by comparing the result of the improved model with the traditional model.
\end{abstract}

Keywords: Power equipment maintenance, probability model, state diagram.

\section{INTRODUCTION}

Components of power equipment may lead to aging and other malfunction problems after running for a long time. The purpose of maintenance is to find and fix the components with hidden faults, and to delay or postpone equipment's aging process, so that equipment failure can be minimized. Successful maintenance can improve the equipment condition, extend its working life and enhance the availability of the equipment.

During the service life of the power equipment, its performance can be effected by various factors. It is difficult to quantify the influence of maintenance on the equipment's life and its reliability by mathematical model [1-4]. If the influence is quantified by taking a deterministic model to make qualitative analysis, the results are often prone to error. Therefore, many researchers have adopted the probability model to simulate the equipment aging process [5-9]. In this paper, an improved state diagram is applied to show the aging process probability model of power equipment. Thus, the relationship between the aging process and multiple states of the system can be represented accurately.

\section{TRADITIONAL STATE DIAGRAM PROBABILITY MODEL}

In the aging process, both natural aging and random factors can lead to equipment's malfunctions. Random failures cannot be prevented effectively. It can be deemed as state transition from normal working state to a random failure state. Random failure probability is roughly constant and is independent of current state of the equipment.

\footnotetext{
*Address correspondence to this author at the Key Laboratory of Power System Intelligent Dispatch and Control of Ministry of Education, Shandong University, Jingshi Road, Jinan City 250061, China; Tel: +86-531-88399003; E-mail: wangyantao@gmail.com
}

The state diagram model is used usually to represent the system aging, inspection and maintenance state. The natural aging process of equipment is composed of several continuously varying states. The transfer process and relations between each state are shown in Fig. (1). Equipment in serious state $\left(S_{3}\right)$ would change into fault state $(F)$, when it had no timely maintenance. If the equipment fails, corrective maintenance can restore it back to its original state. In addition, the equipment in any state can be restored back to the better state when planned maintenance is implemented according to relevant strategies.

If random failure is not considered, it is assumed that maintenance can restore the equipment back to a previous state of the aging process. The transfer process and relations between each state are shown in Fig. (1).

In Fig. (1), the aging process of equipment is represented in three separate states, which are $S_{1} \sim S_{3}$ respectively. If there is no repair after the last aging state, the equipment will be in a failed state which is represented by $F$. After failed state, the system can be restored to state $S_{1}$ through equipment replacement or repair. $M_{1} \sim M_{3}$ represent that the equipment is in its maintenance state.

In the process of equipment aging, there are not only aging and failed states, but also checking state, maintenance state and decision-making, etc. Most of the maintenance decisions are made on the basis of equipment state inspection. Therefore, it is necessary to add the inspection states $I_{1}, I_{2}$ and $I_{3}$ into the model to represent state inspections, which are shown in Fig. (2).

In Fig. (2), $\lambda_{1} \sim \lambda_{3}$ represent the transition probability between the aging states. $\gamma_{1} \sim \gamma_{3}$ represent the inspection probability of the equipment under different aging statuses. $\delta_{1} \sim \delta_{3}$ 


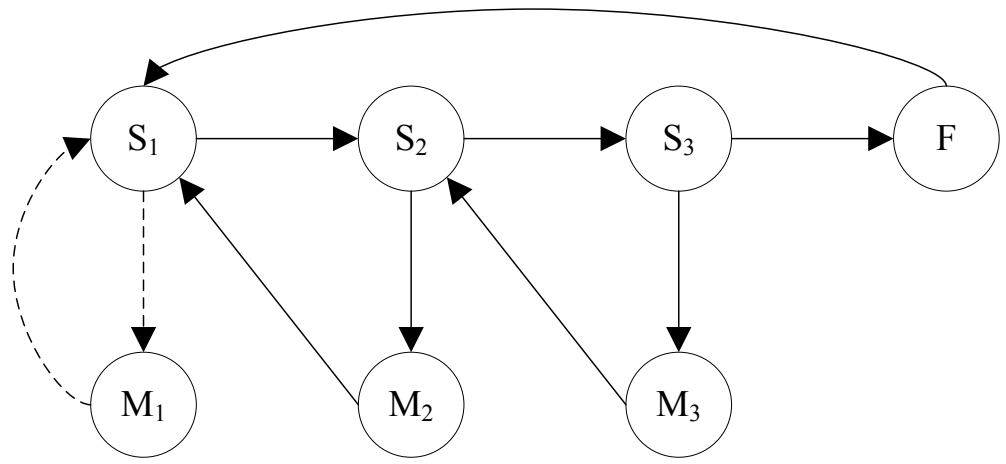

Fig. (1). Equipment aging state diagram model including maintenance.

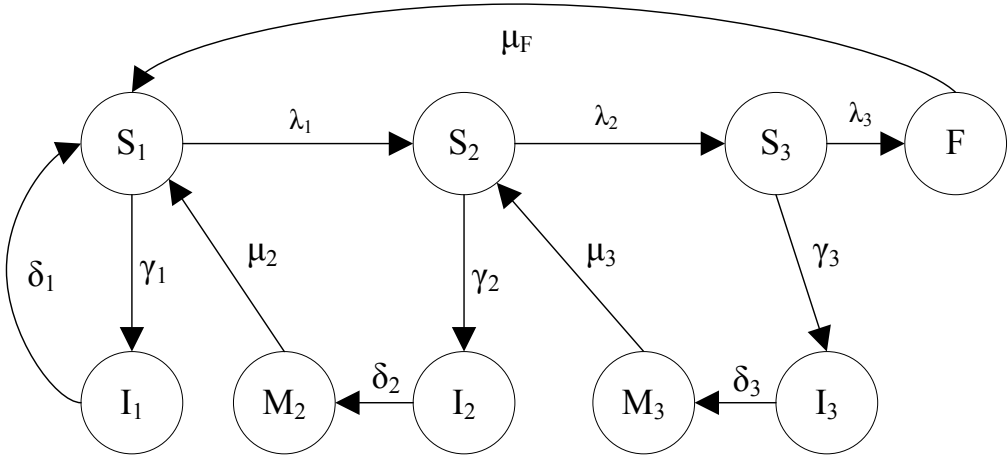

Fig. (2). Equipment aging state diagram model including maintenance and inspection.

represent the maintenance probability after checking. $\mu_{2}$ and $\mu_{3}$ represent the probability that equipment will achieve the maintenance effect after maintenance; and $\mu_{F}$ represents the repair rate that equipment changes from the fault state $F$ to state $S_{1}$.

\section{IMPROVEMENTS IN THE STATE DIAGRAM PROBABILITY MODEL}

\subsection{Cognizance of Equipment State}

Traditional probability model of state diagram describes the basic equipment states in aging process. It can reflect the aging process of equipment and the relationship between aging, inspection and maintenance. However, the model doesn't completely show all the situations, and cannot be used by the maintenance staff in their work. Especially, the cognizance of equipment's current state is lacked during maintenance operation.

In the traditional state diagram model, the inspection frequency is $\gamma_{2}$, if the equipment is in the state $S_{2}$. That is to say, the current state of the equipment is considered to be as known, even if the state inspection has not been taken. In reality when the equipment state turns from $S_{1}$ to $S_{2}$, the maintenance staff can't know until inspection. Therefore, the inspection frequency is still $\gamma_{1}$ and cannot be set to $\gamma_{2}$. If the equipment enters into state $S_{2}$ after maintenance state $M_{3}$, the maintenance staff can know that the current state is $S_{2}$, thus the inspection frequency changes to $\gamma_{2}$.

Therefore, the state graph model can be improved to represent an actual situation as shown in Fig. (3).

In Fig. (3), the degradation states $S_{2}^{\prime}$ and $S_{2}^{\prime \prime}$ represent state $S_{2}$ in the traditional state diagram model; $S_{3}^{\prime}$ and $S_{3}^{\prime \prime}$ represent state $S_{3}$ in the same way. If the system is in the state $S_{1}, S_{2}^{\prime}$ or $S_{3}^{\prime}$, maintenance staff will not know that the equipment has taken state transition without a state inspection. The staff will treat the equipment as it is in state $S_{1}$. Therefore, the inspection frequency should still be kept at $\gamma_{1}$.

Suppose the equipment is in the state $S_{3}^{\prime}$, maintenance staff will make its inspection with frequency $\gamma_{1}$. Only if they 


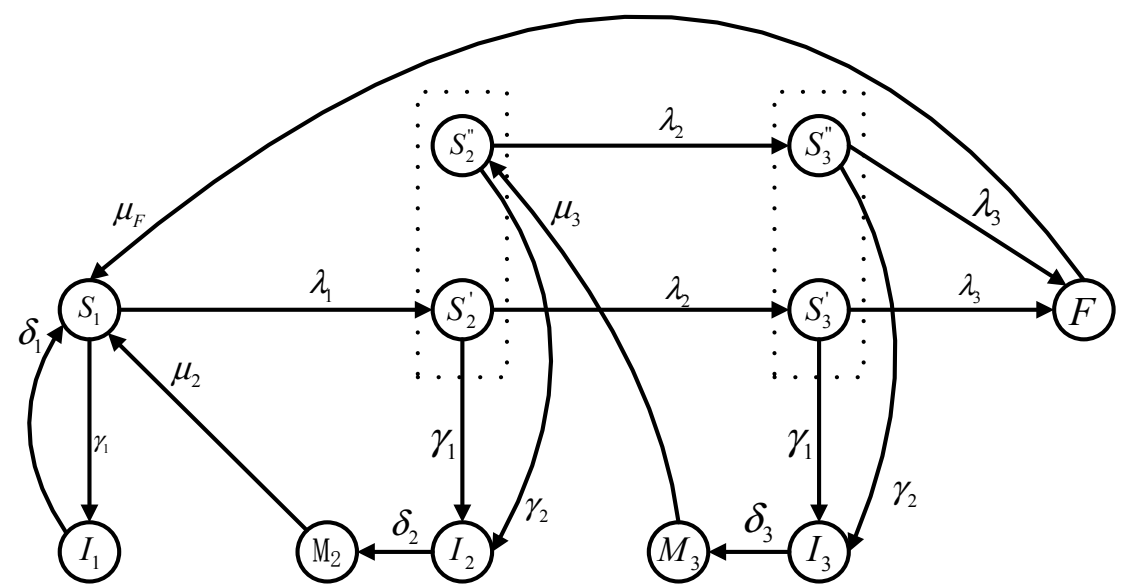

Fig. (3). State diagram probability model considering cognizance.

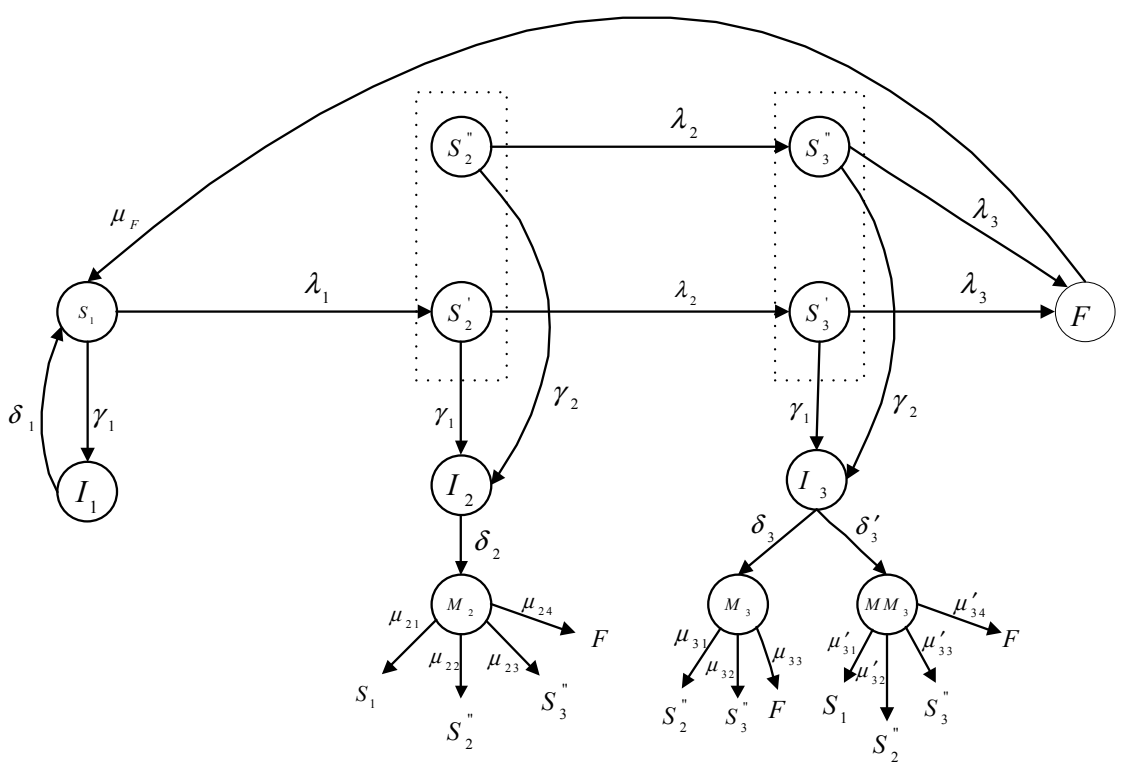

Fig. (4). State diagram probability model considering maintenance decision and effect.

decide to perform maintenance $M_{3}$ and convert the equipment to a new degradation state $S_{2}^{\prime \prime}$, they would know clearly the current state of the equipment and then the inspection frequency would change to $\gamma_{2}$.

\subsection{Maintenance Decision and Effect}

There is an assumption in Fig. (3), that the maintenance must be taken after inspection states $I_{2}$ and $I_{3}$. Both maintenances $M_{2}$ and $M_{3}$ have the same effect, and the equipment must return to the previous state in accordance with the corresponding transformation probability. But maintenance staff should make different maintenance decisions according to the inspection and assessment results acquired from the actual work. The decisions might be to keep in operation minor repairs or overhauls. Maintenance deci- sion will lead to several different results with regards to probability distribution.

Because the states $S_{2}^{\prime}$ and $S_{2}^{\prime \prime}$ belong to the mild aging state, the maintenance decision will be the minor repair $M_{2}$. And the states $S_{3}^{\prime}$ and $S_{3}^{\prime \prime}$ belong to the severe aging state, so the maintenance decision might be minor repair $M_{3}$ or major repair $M M_{3}$. Minor repairs and major repairs have different costs and effects. The improved state graph probability model considering the maintenance decision and effect is shown in Fig. (4).

Based on the analysis results mentioned above, the improved model can describe equipment aging process accurately. And it is convenient to obtain analytical solution if the semi-Markov process is used in reliability analysis. 


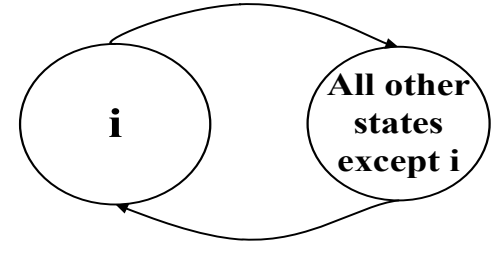

Fig. (5). Two state process system.

\section{SEMI-MARKOV PROCESS AND ITS APPLICA- TION TO THE IMPROVED STATE DIAGRAM PROBABILITY MODEL}

\subsection{Semi-Markov Process}

Electric powered equipment aging is a random process. The equipment running states are transferred between different states according to a certain probability distribution. The state of equipment is uncertain in the future. The aging process can be described by the Markov process and the semiMarkov process [10-13].

If the state transition of the system has a constant frequency, then the future development of the system would not only be related to the current state but will also be related to the past process, and the duration of each state, which would be according to the exponential distribution. That process of state transition can be regarded as a Markov process.

By using the non-aftereffect property, Markov process is suitable for describing the equipment's aging process. Through the mathematical property of Markov process, the reliability index of equipment can be calculated, such as the steady state probability of the equipment in each state, the frequency of the state and the duration of each state. According to the Markov process, the state transition of system is not only related to the current state but also to its past states, so decision makers can make decisions on the basis of the current state information.

Using semi-Markov to describe the state expression and transition of the power transmission and transformation equipment is an improved application of Markov process. SemiMarkov process considers more parameters than Markov process, such as the average residence time of the equipment in each state. Thus, semi-Markov process can be applied in a system which has multiple states and considers the duration of each state. Markov process simplifies the analysis of model and ignores the different duration of the states and only considers the state transitions between the states. In practice, using semiMarkov process is closer to an actual situation.

\subsection{Calculation of Semi-Markov Process}

\section{A. State Stationary Probability}

In the Markov process model, the limit state of system transferred from one state by multiple steps is called the steady state. The steady state probability is a constant, and is not related to the initial state. If the system is in a steady state, the system state probability will maintain stability.
Linear differential equations for the Markov process is

$\frac{d}{d t} p(t)=p(t) A$

$$
A=\left[\begin{array}{cccc}
\lambda_{11} & \lambda_{12} & \cdots & \lambda_{1 n} \\
\lambda_{21} & \lambda_{22} & \cdots & \lambda_{2 n} \\
\vdots & \vdots & \vdots & \vdots \\
\lambda_{n 1} & \lambda_{n 2} & \cdots & \lambda_{n n}
\end{array}\right]
$$
ity.

In formula (2), $\lambda_{i j}$ represents the state transition probabil-

For solving the steady state probability, another equation is required to establish the following linear algebraic equations:

$$
\left\{\begin{array}{c}
P A=0 \\
\sum P_{i}=1
\end{array}\right.
$$

The steady state probability calculated from the above equations can be used to solve other reliability indices.

\section{B. Frequency and Average Duration}

If the system is stable, the average number stays in one state per unit time. This is known as the access frequency in the state. The access frequency and the average duration are important indices for reliability analysis. For the two indices solution, the frequency and duration method [14] should be used to represent the relationships between the state probability, access frequency and the duration of the state. In order to determine the relationships, the time of system in and out of the state should be calculated.

A two state process system is established, as shown in Fig. (5).

$T_{c i}=T_{i}+T_{i}^{\prime}$

In formula (4), $T_{i}$ and $T_{i}^{\prime}$ represent the average time in and out of state $i$, and $T_{c i}$ is the average cycle time.

By definition, access frequency is equal to the reciprocal of the average cycle time.

$f_{i}=\frac{1}{T_{c i}}$

With both sides of the formula (5) multiplied by $T_{i}$ :

$f_{i} T_{i}=\frac{T_{i}}{T_{c i}}$

So, the state probability of $i$ is:

$\frac{T_{i}}{T_{c i}}=p_{i}$ 
Then,

$f_{i}=\frac{1}{T_{c i}}=\frac{T_{i}}{T_{c i}} \times \frac{1}{T_{i}}=\frac{p_{i}}{T_{i}}$

The transfer frequency $f_{i j}$ refers to the expectation times of system transferred from state $i$ to $j$ directly per unit time.

$$
\begin{aligned}
f_{i j} & =\lim _{\Delta t \rightarrow 0} \frac{1}{\Delta t} p\{[X(t+\Delta t)=j \cap X(t)=i]\} \\
& =\lim _{\Delta t \rightarrow 0} \frac{1}{\Delta t} p\{[X(t+\Delta t)=j \mid X(t)=i] p[X(t)=i]\} \\
& =\lim _{\Delta t \rightarrow 0} \frac{1}{\Delta t}\left(\lambda_{i j} \Delta t\right) p[X(t)=i]=\lambda_{i j} p_{i}
\end{aligned}
$$

Also, the access frequency of state $i$ amounts to the sum of all transfer frequency from state $i$ to the other state.

$f_{i}=\sum_{i \neq j} f_{i j}$

Put the formula (10) into the formula (9),

$f_{i}=\sum_{i \neq j} f_{i j}=p_{i} \sum_{j \neq i} \lambda_{i j}=\sum_{j \neq i} p_{j} \lambda_{j i}$

When the system is stable, the transfer frequency into and out of state $i$ is equal. It is the frequency equilibrium.

Then, the average duration of state $i$ is,

$$
\mathrm{T}_{i}=\frac{p_{i}}{f_{i}}=\frac{1}{\sum_{i \neq j} \lambda_{i j}}
$$

The formula (12) means that the average duration of a given state is equivalent to the reciprocal of the sum of each probability that the state is transferred into.

\section{SIMULATION AND RESULT ANALYSIS}

A classical case is selected to calculate the steady state probability of the circuit breaker, the average duration and the access frequency of each state. The calculation results are compared, which is based on the traditional state diagram probability model, the improved model and Monte Carlo simulation respectively.

Monte Carlo simulation is a mathematical method to calculate the distribution characteristics of the time series by setting random process, generating time series repeatedly, and then calculating parameter estimator and statistics number.

In reliability analysis and design, Monte Carlo simulation can determine the probability distribution and digital characteristics of complicated random variables. Its advantage is that the prediction accuracy is gradually increased along with the increase of simulation number. And its disadvantage is that it spends a lot of time to generate the time sequence. If the case [9] is simplified, it can get high accuracy results while calculating with Monte Carlo simulation and the result

$P(n)=\left[\begin{array}{cccccccccccc}1-\lambda_{1}-\gamma_{1} & \lambda_{1} & 0 & 0 & 0 & 0 & \gamma_{1} & 0 & 0 & 0 & 0 & 0 \\ 0 & 1-\lambda_{2}-\gamma_{1} & 0 & \lambda_{2} & 0 & 0 & 0 & \gamma_{1} & 0 & 0 & 0 & 0 \\ 0 & 0 & 1-\lambda_{2}-\gamma_{2} & 0 & 0 & 0 & 0 & \gamma_{2} & 0 & 0 & 0 & 0 \\ 0 & 0 & 0 & 1-\lambda_{3}-\gamma_{1} & \lambda_{2} & \lambda_{3} & 0 & 0 & 0 & \gamma_{1} & 0 & 0 \\ 0 & 0 & 0 & 0 & 1-\lambda_{3}-\gamma_{2} & \lambda_{3} & 0 & 0 & 0 & \gamma_{2} & 0 & 0 \\ \mu_{\mathrm{F}} & 0 & 0 & 0 & 0 & 1-\mu_{\mathrm{F}} & 0 & 0 & 0 & 0 & 0 & 0 \\ \delta_{1} & 0 & 0 & 0 & 0 & 0 & 1-\delta_{1} & 0 & 0 & 0 & 0 & 0 \\ 0 & 0 & 0 & 0 & 0 & 0 & 0 & 1-\delta_{2} & \delta_{2} & 0 & 0 & 0 \\ \mu_{21} & 0 & \mu_{22} & 0 & \mu_{23} & \mu_{24} & 0 & 0 & 1-\mu_{21}-\mu_{22}-\mu_{23}-\mu_{24} & 0 & 0 & 0 \\ 0 & 0 & 0 & 0 & 0 & 0 & 0 & 0 & 0 & 1-\delta_{3}-\delta_{3}^{\prime} & \delta_{3} & 0 \\ 0 & 0 & \mu_{31} & 0 & \mu_{32} & \mu_{33} & 0 & 0 & 0 & 0 & 1-\mu_{31}-\mu_{32}-\mu_{33} & 0 \\ \mu_{31}^{\prime} & 0 & \mu_{32}^{\prime} & 0 & \mu_{33}^{\prime} & \mu_{34}^{\prime} & 0 & 0 & 0 & 0 & 0 & 1-\mu_{31}^{\prime}-\mu_{32}^{\prime}-\mu_{33}^{\prime}-\mu_{34}^{\prime}\end{array}\right]$

By formula (3),

$$
\left.P(n)=\left[\begin{array}{l}
P_{0} \\
P_{1} \\
P_{2} \\
P_{3} \\
P_{4} \\
P_{5} \\
P_{6} \\
P_{7} \\
P_{8} \\
P_{9} \\
P_{10} \\
P_{11}
\end{array}\right]=\left[\begin{array}{cccccccccccc}
1 & 1 & 1 & 1 & 1 & 1 & 1 & 1 & 1 & 1 & 1 & 1 \\
\lambda_{1} & -\lambda_{2}-\gamma_{1} & 0 & 0 & 0 & 0 & 0 & 0 & 0 & 0 & 0 & 0 \\
0 & 0 & -\lambda_{2}-\gamma_{2} & 0 & 0 & 0 & 0 & 0 & \mu_{22} & 0 & \mu_{31} & \mu_{32}^{\prime} \\
0 & \lambda_{2} & 0 & -\lambda_{3}-\gamma_{1} & 0 & 0 & 0 & 0 & 0 & 0 & 0 & 0 \\
0 & 0 & \lambda_{2} & 0 & -\lambda_{3}-\gamma_{2} & 0 & 0 & 0 & \mu_{23} & 0 & \mu_{32} & \mu_{33}^{\prime} \\
0 & 0 & 0 & \lambda_{3} & \lambda_{3} & -\mu_{\mathrm{F}} & 0 & 0 & \mu_{24} & 0 & \mu_{33} & \mu_{34}^{\prime} \\
\gamma_{1} & 0 & 0 & 0 & 0 & 0 & -\delta_{1} & 0 & 0 & 0 & 0 & 0 \\
0 & \gamma_{1} & \gamma_{2} & 0 & 0 & 0 & 0 & -\delta_{2} & 0 & 0 & 0 & 0 \\
0 & 0 & 0 & 0 & 0 & 0 & 0 & \delta_{2}-\mu_{21}-\mu_{22}-\mu_{23}-\mu_{24} & 0 & 0 & 0 \\
0 & 0 & 0 & \gamma_{1} & \gamma_{2} & 0 & 0 & 0 & 0 & -\delta_{3}-\delta_{3}^{\prime} & 0 & 0 \\
0 & 0 & 0 & 0 & 0 & 0 & 0 & 0 & 0 & \delta_{3} & -\mu_{31}-\mu_{32}-\mu_{33} & 0 \\
0 & 0 & 0 & 0 & 0 & 0 & 0 & 0 & 0 & \delta_{3}^{\prime} & 0 & -\mu_{31}^{\prime}-\mu_{32}^{\prime}-\mu_{33}^{\prime}-\mu_{34}^{\prime}
\end{array}\right]\right]^{-1}\left[\begin{array}{c}
1 \\
0 \\
0 \\
0 \\
0 \\
0 \\
0 \\
0
\end{array}\right]_{0}
$$


Table 1. Value of state transition probability.

\begin{tabular}{|c|c|c|c|}
\hline Transition Probability & $\begin{array}{c}\text { Value } \\
(1 / \text { year })\end{array}$ & Transition Probability & $\begin{array}{c}\text { Value } \\
(1 / \text { year) }\end{array}$ \\
\hline \hline$\lambda_{1}$ & 0.33 & $\mu_{21}$ & 330 \\
\hline$\lambda_{2}$ & 0.29 & $\mu_{22}$ & 10 \\
\hline$\lambda_{3}$ & 0.5 & $\mu_{23}$ & 10 \\
\hline$\gamma_{1}$ & 0.5 & $\mu_{24}$ & 340 \\
\hline$\gamma_{2}$ & 1 & $\mu_{31}$ & 10 \\
\hline$\gamma_{3}$ & 1 & $\mu_{32}$ & 10 \\
\hline$\delta_{1}$ & 360 & $\mu_{33}$ & $\mu_{31}^{\prime}$ \\
\hline$\delta_{2}$ & 360 & $\mu_{32}^{\prime}$ & 170 \\
\hline$\delta_{3}$ & 180 & $\mu_{33}^{\prime}$ & 10 \\
\hline$\delta_{3}^{\prime}$ & 180 & $\mu_{34}^{\prime}$ & 10 \\
\hline
\end{tabular}

Table 2. State stationary probability.

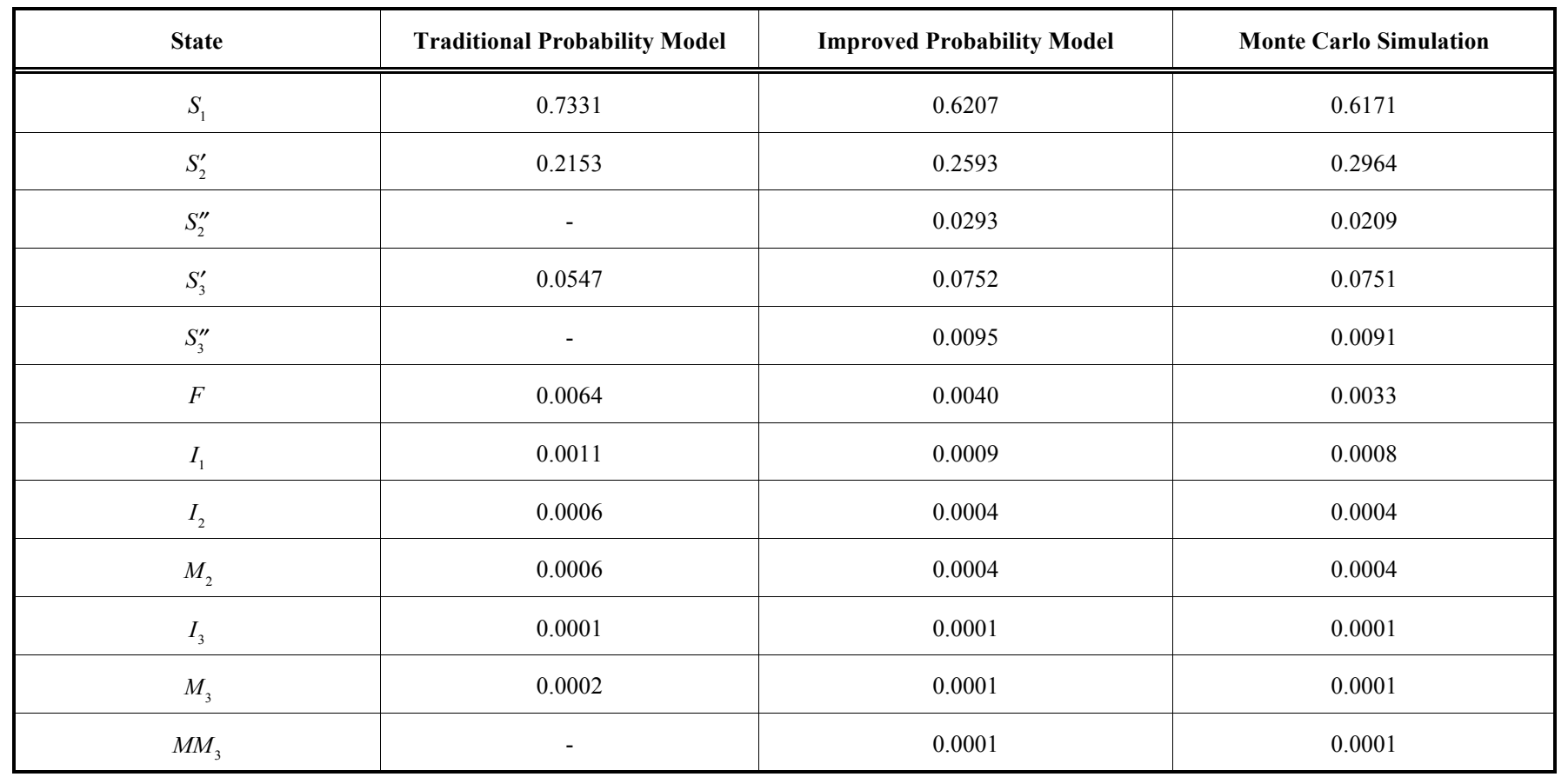

is adaptive enough to be the basic value of comparison between the traditional state diagram probability model and the improved model.

The state transition probabilities are as listed in Table $\mathbf{1 .}$

Suppose the state probabilities of $S_{1}, S_{2}^{\prime}, S_{2}^{\prime \prime}, S_{3}^{\prime}, S_{3}^{\prime \prime}$, $F, I_{1}, I_{2}, M_{2}, I_{3}, M_{3}, M M_{3}$ are $P_{0}, P_{1}, P_{2}, P_{3}, P_{4}, P_{5}$, $P_{6}, P_{7}, P_{8}, P_{9}, P_{10}, P_{11}$ respectively.
The state transition matrix is,

Substituting the data in Table $\mathbf{1}$, the reliability indices acquired through three methods are shown in Table 2, Table 3 and Table 4 respectively.

It can be seen that compared with the traditional state diagram probability model, the improved model has closer results to the Monte Carlo simulation. The improved state diagram probability model is more practical to be applied to 
Table 3. Access frequency.

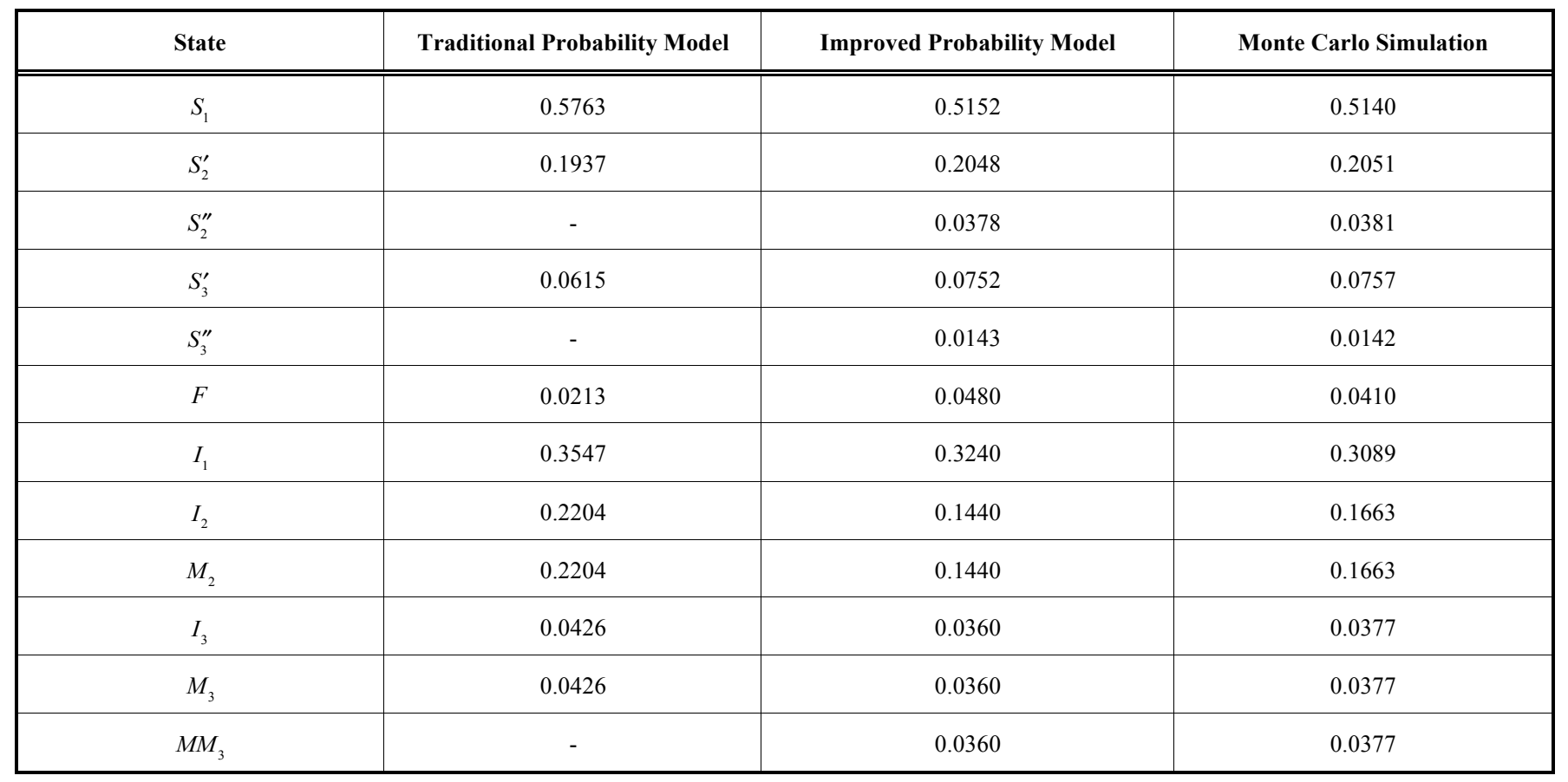

Table 4. Average duration.

\begin{tabular}{|c|c|c|c|}
\hline State & Traditional Probability Model & Improved Probability Model & Monte Carlo Simulation \\
\hline$S_{1}$ & 1.2072 & 1.2048 & 1.200 \\
\hline$S_{2}^{\prime \prime}$ & - & 0.7752 & 0.754 \\
\hline$S_{3}^{\prime \prime}$ & - & 0.6667 & 0.633 \\
\hline$F$ & 0.0833 & 0.0833 & 0.083 \\
\hline$I_{1}$ & 0.0028 & 0.0028 & 0.003 \\
\hline$I_{2}$ & 0.0028 & 0.0028 & 0.003 \\
\hline$M_{3}$ & 0.0028 & 0.0028 & 0.003 \\
\hline$M M_{3}$ & - & 0.0028 & 0.003 \\
\hline
\end{tabular}

simulate the aging and maintenance process of circuit breaker. The validity of the improved model is proved.

\section{CONCLUSION}

Maintenance is one of the important tasks of power equipment management. The premise of making maintenance strategy is to have a clear understanding of the aging process and to forecast the maintenance effect and the future operation of the equipment accurately. Modeling the aging and maintenance process with state diagram probability model is in accordance with the laws and characteristics of equipment aging. By analyzing the traditional state diagram probability model and amending the unconformities to the actual maintenance, the improved state diagram probability model is more reasonable to represent the selection of the state monitoring frequency in practice. The improved model is closer to the actual situation. As a result the need of elec- 
trical equipment maintenance task can be satisfactorily fulfilled.

\section{CONFLICT OF INTEREST}

The authors confirm that this article content has no conflict of interest.

\section{ACKNOWLEDGEMENTS}

This study is supported by National Natural Science Foundation of China (No.51077087 and No.51007047).

\section{REFERENCES}

[1] J. Endrenyi, G. Anders, and A. Leite da Silva, "Probabilistic evaluation of the effect of maintenance on reliability-an application", IEEE Transactions on Power Systems, vol. 13, pp. 576-583, 1998.

[2] Z. Lu, H. Huang, and C. Fu, "Exploration on life evaluation model of power transformer based on state evaluation technology", East China Electric Power, vol. 37, pp. 1019-1022, 2009.

[3] M. Li, X. Han, M. Yang, and Z. Guo, "Studies on power grid maintenance concept and theoretical basis", In: Proceedings of the Chinese Society for Electrical Engineering, vol. 31, pp. 43-52, 2011.

[4] J. Endrenyi, S. Aboresheid, and R. Allan, "The present status of maintenance strategies and the impact of maintenance on reliability", IEEE Transactions on Power Systems, vol. 16, pp. 638-646, 2001.
[5] F. Camci, "System maintenance scheduling with prognostics information using genetic algorithm", IEEE Transactions on Reliability, vol. 58, pp. 539-552, 2009.

[6] R. Liao, Q. Wang, S. Luo, Y. Liao, and C. Sun, "Evaluation model of power transformer running state based on fuzzy comprehensive evaluation", Automation of Electric Power Systems, vol. 32, pp. 7075, 2008.

[7] Z. Yang, D. Djurdjanovic, and J. Ni, "Maintenance scheduling in manufacturing systems based on predicted machine degradation", Journal of Intelligent Manufacturing, vol. 19, pp. 87-98, 2008.

[8] M. Li, X. Han, Y. Wang, Z. Guo, and G. Liu, "Decision-making model and the solution of substation maintenance state", In: Proceedings of the Chinese Society for Electrical Engineering, vol. 32, pp. 196-202, 2012.

[9] T. M. Welte, "Using state diagrams for modeling maintenance of deteriorating systems", IEEE Transactions on Power Systems, vol. 24, pp. 58-66, 2009.

[10] M. L. Puterman, Markov Decision Processes: Discrete Stochastic Dynamic Programming, New York: John Wiley and Sons Inc., 1994.

[11] F. Yang, C.M. Kwan, and C.S. Chang, "Multi-objective evolutionary optimization of substation maintenance using decision-varying Markov Model", IEEE Transactions on Power Systems, vol. 23, pp. 1328-1335, 2008.

[12] H. Ge, and S. Asgarpoor, "Reliability evaluation of equipment and substations with fuzzy Markov Processes", IEEE Transactions on Power Systems, vol. 25, pp. 1319-1328, 2010.

[13] C. S. Liu, J. Ge, and Y. H. Shen, "Maintenance decision of generator state based on Markov Chain", Power System and Automation, vol. 18, pp. 82-85, 2006

[14] Y. Guo, The Theory of Reliability Engineering, Beijing: Tsinghua University press, 2002.

Received: December 15, 2014

(C) Wang and Han; Licensee Bentham Open.

This is an open access article licensed under the terms of the Creative Commons Attribution Non-Commercial License (http://creativecommons.org/licenses/by$\mathrm{nc} / 4.0 /$ ) which permits unrestricted, non-commercial use, distribution and reproduction in any medium, provided the work is properly cited. 